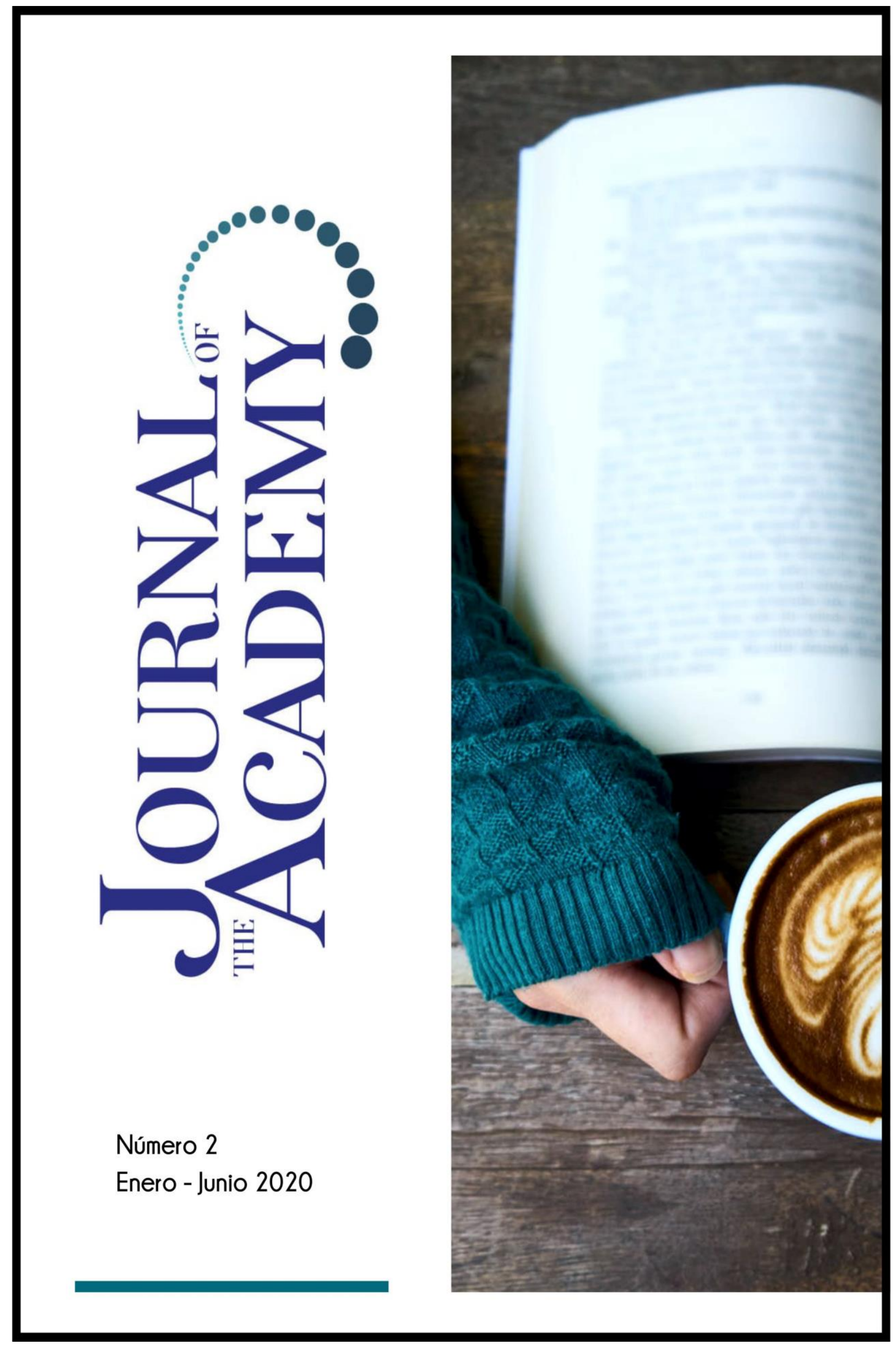




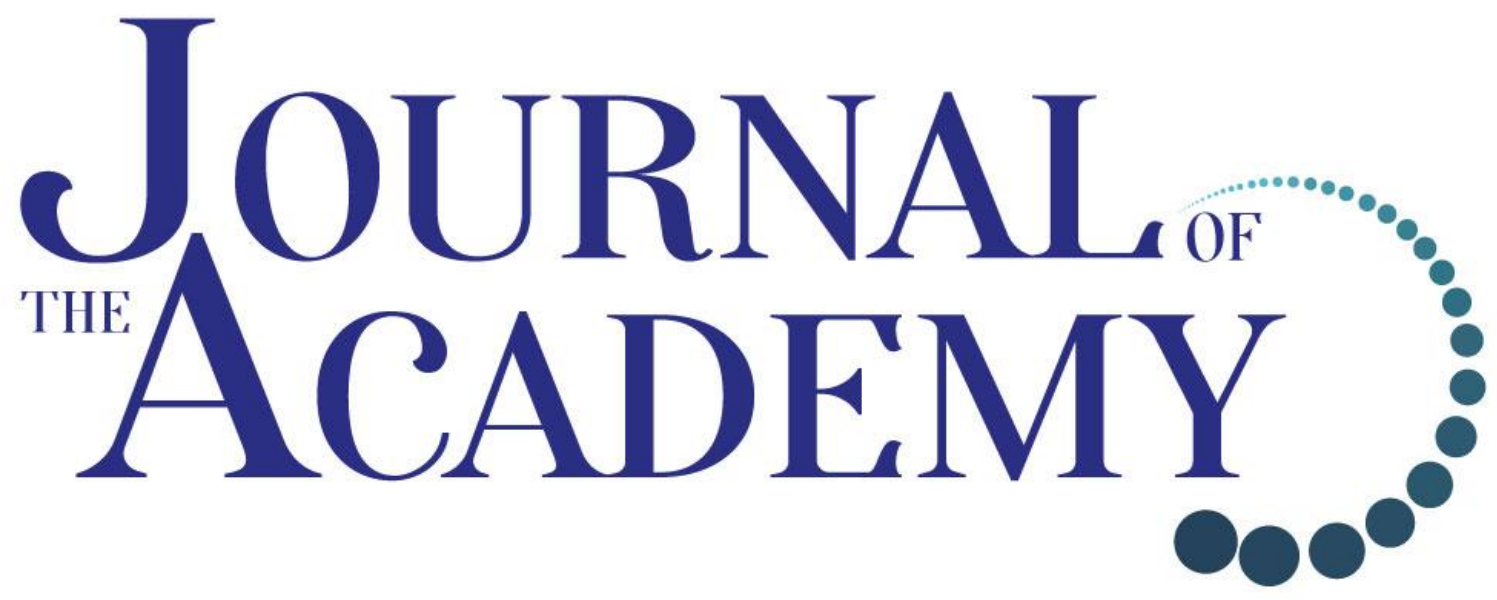

Publicación Científica de la Asociación de Universidades del Perú ASUP

Número 2

Enero-Junio 2020

www.journalacademy.org 


\section{ISSN 2707-0301}

\section{Directora-Editora en Jefe}

Ada Gallego Ruiz Conejo

Editor Científico

Oswaldo Orellana Manrique

Comité Científico

\section{Javier Carreón Guillén}

Universidad Nacional Autónoma de México, México Martino Contu

Universidad de Sassari, Italia

Jorge Enrique Elías Caro

Universidad del Magdalena, Colombia

Roberto Escalante Semerena

Universidad Nacional Autónoma de México, México

Oscar Ortega Arango

Universidad Autónoma de Yucatán, México

Alex Veliz Burgos

Universidad de Los Lagos, Chile

Comité Editorial

\section{Manuela Garau}

Centro Studi Sea, Italia

José Manuel González Freire

Universidad de Colima, México

Carlos Tulio da Silva Medeiros

Diálogos en Mercosur, Brasil

Eduardo Gomes Onofre

Universidade Estadual da Paraíba, Brasil

\section{Equipo Ejecutivo}

Juan Carlos Norabuena Castañeda

Thalia Chávez Cortéz

Journal Academy

Revista Semestral Open Access 


\title{
EXPERIENCIA EN LA MEJORA DE LAS HABILIDADES SOCIALES EN ESTUDIANTES DE EDUCACIÓN SECUNDARIA: PADRE SUSTITUTO, PROVINCIA ANDAHUAYLAS, PERÚ
}

\section{EXPERIENCE IN THE IMPROVEMENT OF SOCIAL SKILLS IN SECONDARY EDUCATION STUDENTS: PADRE SUBSTITUTE, ANDAHUAYLAS PROVINCE, PERU}

Recibido: 23 de julio de 2019

Aceptado: 20 de noviembre de 2019

\author{
William Camilo YAURIS POLO \\ Universidad Nacional José María Arguedas, Perú \\ wyauris@gmail.com \\ Felipe Rafael VALLE DÍAZ \\ Universidad Nacional José María Arguedas, Perú \\ Yudy PÉREZ ZÚÑIGA \\ Universidad Nacional José María Arguedas, Perú \\ wyauris@gmail.com
}

\begin{abstract}
The purpose of the research was to implement a program of talks to strengthen social skills in high school students by complementing the figure of the surrogate parent. The main problem is students who do not have parents, others who do not recognize them and some who do not live with their parents, in addition to being exposed to social risks. The research is of the applied type, whose design was preexperimental. The population was 765 students of the Agricultural Educational Institution No. 08.
\end{abstract}

Keywords: social skill, communication, assertiveness, decision making

Resumen: La investigación tuvo como finalidad implementar un programa de charlas para fortalecer las habilidades sociales en estudiantes de secundaria a partir de complementar la figura del padre sustituto. El problema primordial son estudiantes que no tienen progenitores, otros que no los reconocen y algunos que no viven con sus progenitores, además de estar expuestos a riesgos sociales. La investigación es del tipo aplicada, cuyo diseño fue pre experimental. La población fueron 765 estudiantes de la Institución Educativa Agropecuario $\mathrm{N}^{\circ} 08$.

Palabras claves: clave: habilidad social, comunicación, asertividad, toma de decisiones

\section{Para Citar este Artículo:}

Yauris Polo, William Camilo; Valle Díaz, Felipe Rafael y Pérez Zúñiga, Yudy. Experiencia en la mejora de las habilidades sociales en estudiantes de educación secundaria: Padre Sustituto, Provincia Andahuaylas, Perú. Journal of Academy num 1 (2019): 11-19.

Licencia Creative Commons Atributtion Nom-Comercial 3.0 Uunported

(CC BY-NC 3.0)

Licencia Internacional

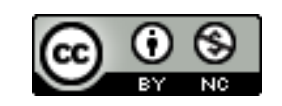




\section{Introducción}

En el presente trabajo de investigación se hablará y profundizará sobre el nivel de habilidades sociales de los estudiantes y el padre sustituto. Tanto así muchos estudiantes afirman que tienen muchos problemas que no pueden salir de ellos y frenan ser exitosos, impidiendo lograr colocar sus vidas al nivel en el que sueñan. En cierta medida, no todos los estudiantes cuentan con las mismas oportunidades, el mismo nivel de habilidades sociales, la misma preparación intelectual ni la misma base económica.

La autoestima, es el sentimiento valorativo de nuestro ser, de nuestra manera de sentir y ser, de quienes somos nosotros y ellos, del conjunto de rasgos corporales, mentales y espirituales que configuran nuestra personalidad, esta se aprende, cambian y la podemos mejorar en todo momento.

Dado el nivel en que se encuentra la autoestima de las personas, esta es responsable de muchos fracasos y éxitos, ya que una autoestima apropiada y buena, muestra y está vinculada a un concepto positivo o negativo de uno mismo y de lo uno mismo se puede concebir, potenciar la capacidad de las personas para desarrollar sus habilidades sociales y aumentar el nivel de seguridad integral y personal, mientras que una habilidad social inadecuada enfocará y se mostrara a la persona hacia la derrota y el fracaso personal y las consecuencias que estas traen al no desarrollarlas adecuadamente.

lannizzotto, (2009) refiere: Los sentimientos de autoestima en particular, y la felicidad en general, se desarrollan como efectos secundarios: de superar los problemas y las adversidades que en la vida se presenta, de realizar las tareas con éxito, de vencer la frustración y el aburrimiento, y sobre todo de obrar y de hacer el bien. El sentimiento de autoestima es un efecto de hacer las cosas bien y de hacer el bien. $Y$ una vez que ésta se ha asentado en el niño de manera positiva, mueve a nuevos esfuerzos, en búsqueda de nuevos logros (p.103).

Conducta no habilidosa ej. Decir una bobada o anti-sociales (ej. golpear a alguien) puede de hecho ser reforzadas según Arkowitz, 1981, Rakos, 1983, el efecto de hacer bien las cosas en la interrelación y el actuar de cada uno como refiere: Emmons y Alberti 1983.

Por entonces la habilidad social es utilizada desde una perspectiva distinta empieza a utilizarse como sustituta de conducta asertiva, o esta última queda englobada en la primera en irse ampliando el concepto. Ambos términos se han utilizado hace mucho tiempo de forma intercambiable

Habilidades sociales en término que es utilizado en el presente trabajo de investigación la cual como mencionaron los autores anteriormente y lo utilizamos en el presente trabajo para medir las habilidades de los estudiantes y que estos de qué manera afectan e incluyen en el desarrollo de los estudiantes.

\section{Desarrollo}

\section{Tipo de investigación}

El tipo de investigación es aplicada, ya que sirve para resolver problemas; asimismo, es de enfoque cuantitativa. Hernández, Fernández y Baptista (2006) nos dicen que "el investigador recolecta datos numéricos de los objetos, fenómenos o participantes, que estudia y analiza mediante procedimientos estadísticos" (p. 5).

\section{Métodos de Investigación}

El método es el descriptivo porque consiste en describir, analizar e interpretar sistemáticamente un 
conjunto de hechos o fenómenos y sus variables que les caracterizan de manera tal como se dan, según Sánchez Carlessi, (2006 p.50).

\section{Diseño de investigación}

El diseño de la investigación es experimental, ya que existe manipulación activa de alguna variable tal como refiere el autor "Es la que se realiza sin manipular deliberadamente variables", según Hernández, et al. 2006 p.48. El diseño de la presente investigación es:

\section{$\mathrm{M}, \mathrm{m1}, 01 \mathrm{~T} 02$}

Dónde: M: Población general, m: muestra escogida de 60 estudiantes, con problema identificado, 01: Nos indica el estado inicial previo diagnóstico, O2: Resultado producto del tratamiento, T: tratamiento de seguimiento a los 60 estudiantes.

El experimento se procede: Por decisión y consenso de la comisión se hace una capacitación y sensibilización general a todos los estudiantes de la Institución Educativa Agropecuario $N^{\circ} 08$. Después de esta capacitación general; se identificó y seleccionó los casos que existen con problemas moderados a fuertes, siendo estos 60 estudiantes, los cuales reciben charlas y tratamiento personalizado.

\section{Población}

Según Sánchez, H. (1994 p.98). La población comprende a todos los miembros de cualquier clase bien definida de personas, eventos u objetos. La población estuvo constituida por 765 estudiante de $1^{\circ}$ a $5^{\circ}$ grado de la Institución Educativa Secundaria Agropecuario Nº 08. Fuente: ESCALE y IE 08, 2017.

\section{Muestra}

La muestra se considera censal pues se seleccionó del $100 \%$ de la población al considerarla un número manejable de sujetos en forma inicial. En este sentido Ramírez (1997) establece la muestra censal es aquella donde todas las unidades de investigación son consideradas como muestra. Los identificados y seleccionados fueron 60 estudiantes del colegio.

\section{Operacionalización}

Según la Organización Mundial de la Salud (OMS), una habilidad social presenta los siguientes aspectos, que puede alinearse como indicadores, siendo estos: asertividad. comunicación, autoestima, toma de decisiones. La escala de valoración (baremo) fue adaptada a partir de la ficha técnica: 2005 Perú variable a trabajar. Es reconocido el baremo siguiente: baja habilidad social (menor a 125 puntos), habilidad social promedio baja (entre 126 a 140 puntos), habilidad social promedio (entre 141 a 150 puntos), habilidad social promedio alta (entre 151 a 160 puntos) y alta habilidad social (mayor a 161 puntos).

\section{Resultados}

\section{Tabla $\mathbf{N}^{\circ} \mathbf{1}$}

Niveles de habilidad social general

\begin{tabular}{|c|c|c|}
\hline & Niveles & Frecuencia \\
\hline & Muy alto (174 a mas) & 12 \\
\hline & Alto (161 a 172 ) & 98 \\
\hline
\end{tabular}



3. Promedio alto (151 a 160)
4. Promedio (141 a 150)
5. Promedio bajo (126 a 140)
6. Bajo (88-125)
7. Muy bajo (Menor a 88)

En los estudiantes de secundaria de la Institución Educativa Agropecuario 08 con Jornada Escolar Completa, se puede observar que la mayor cantidad de estudiantes está representado por 98 nivel alto, en el nivel muy bajo se encuentran 15, en el nivel promedio alto está representado por 255 y en el nivel promedio bajo se encuentran un grupo de 134 lo que significa que hay estudiantes con nivel de habilidad social bajo o nivel de habilidad social inadecuadas.

\section{HABILIDAD SOCIAL}

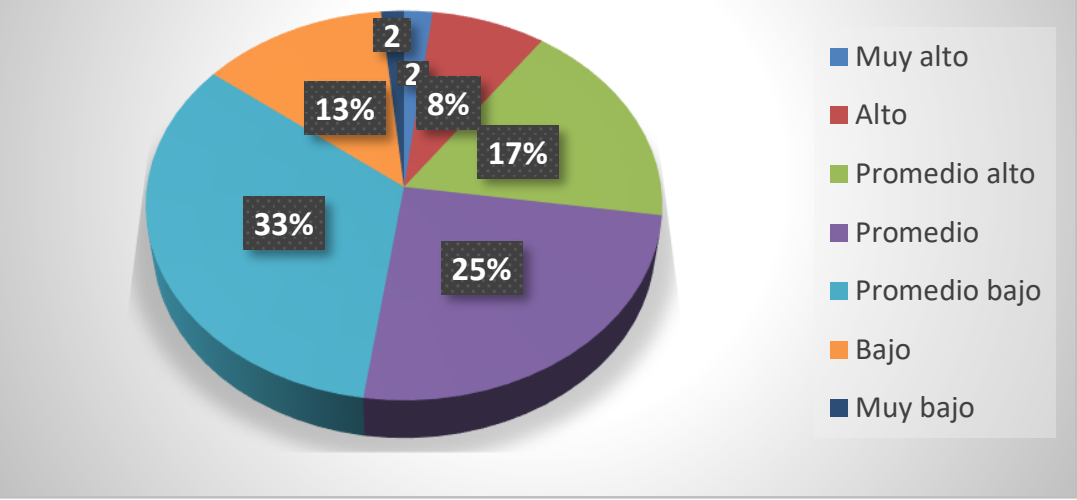

Figura $\mathbf{N}^{\circ} 1$

Comentario: En los estudiantes de secundaria de la Institución Educativa Agropecuario 08 con Jornada Escolar Completa, se puede observar que el mayor porcentaje es $33 \%$ que significa un nivel de habilidades sociales promedio bajo, el $17 \%$ de estudiantes representa el nivel de habilidad promedio alto, el $25 \%$ de estudiantes representa el nivel de habilidad social promedio, el $13 \%$ de estudiantes representa el nivel de habilidad social bajo y el $08 \%$ que significa el nivel de autoestima alto, con la cual observamos que el mayor porcentaje de los estudiantes muestran una habilidad social promedio bajo y bajo, $48 \%$ de habilidades sociales inadecuadas.

\section{Tabla $\mathbf{N}^{\circ} 2$}

Frecuencia dimensión de autoestima

\begin{tabular}{ll}
\hline Niveles & Frecuencia \\
\hline 1. Muy bajo (Menor a 21) & 08 \\
2. Bajo (21 a 34) & 97 \\
3. Promedio bajo (35 a 41) & 266 \\
4. Promedio (42 a 46) & 187 \\
5. Promedio alto (47 a 50) & 132 \\
6. Alto (51 a 54) & 63 \\
7. Muy Alto (55 a mas) & 12 \\
\hline
\end{tabular}

- En la frecuencia de todos los factores, se puede observar la habilidad social en su dimensión autoestima la mayor cantidad de estudiantes está representado por 266 promedio bajo, siguiente un nivel promedio 187, el nivel promedio alto 132, el nivel bajo 97 y el nivel muy bajo con 08 estudiantes. 


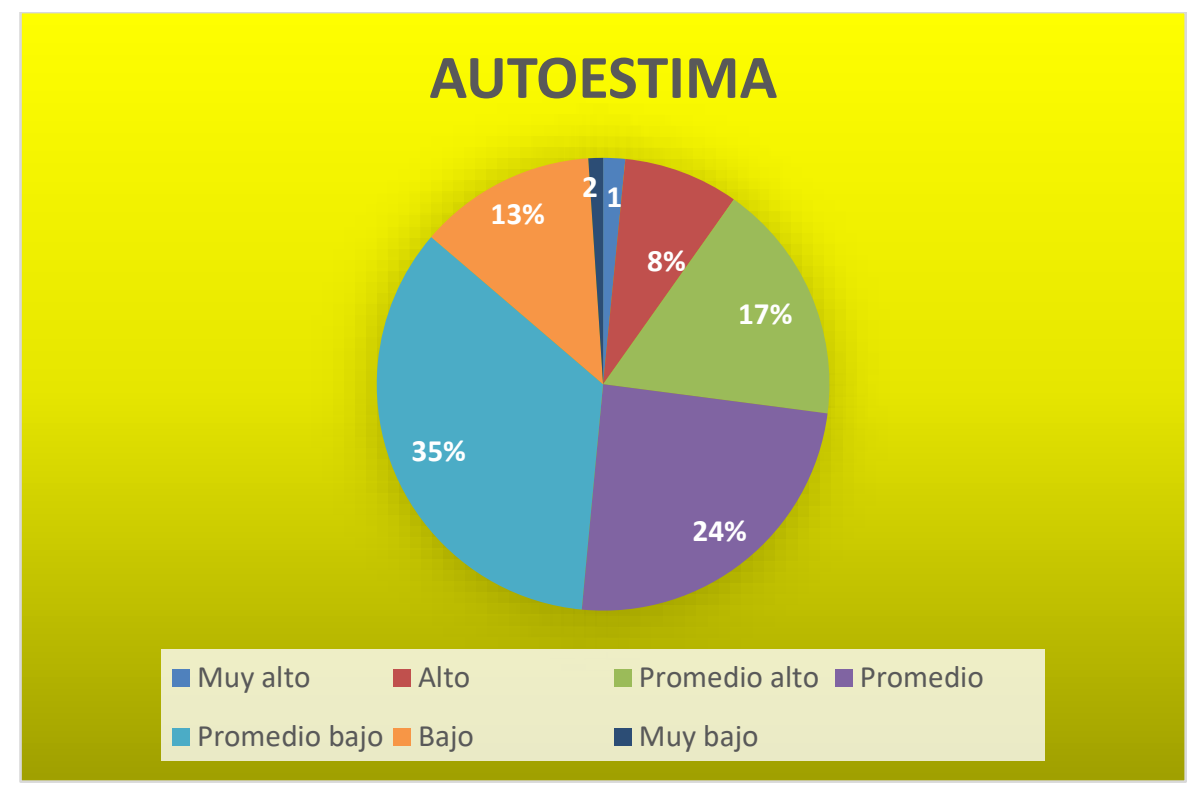

\section{Figura 02}

Comentario: La habilidad social en su dimensión autoestima, se puede observar que el mayor porcentaje es $35 \%$ que significa una autoestima promedio bajo, el $24 \%$ de los estudiantes representa una autoestima de nivel promedio, el $17 \%$ de los estudiantes representa una autoestima de nivel promedio alto, el $01 \%$ de los estudiantes representa una autoestima de nivel muy alto y el nivel de autoestima muy bajo el $02 \%$ de los estudiantes

\section{Tabla $\mathbf{N}^{\circ} 3$}

Frecuencia toma de decisiones

\begin{tabular}{ll} 
Niveles & Frecuencia \\
\hline 1. Muy bajo (Menor a 16) & 03 \\
2. Bajo (16 a 24) & 103 \\
3. Promedio bajo (25 a 29) & 303 \\
4. Promedio (30 a 33) & 188 \\
5. Promedio alto (34 a 36) & 132 \\
6. Alto (37 a 40) & 35 \\
7. Muy Alto (41 a más) & 01 \\
\hline
\end{tabular}

- En la frecuencia de todos los factores, se puede observar la habilidad social en su dimensión toma de decisiones la mayor cantidad de estudiantes está representado por 303 promedio bajo, siguiente un nivel promedio 188, el nivel promedio alto 132, el nivel bajo 103 y el nivel muy bajo con 03 estudiantes. 


\section{TOMA DE DECISIONES}

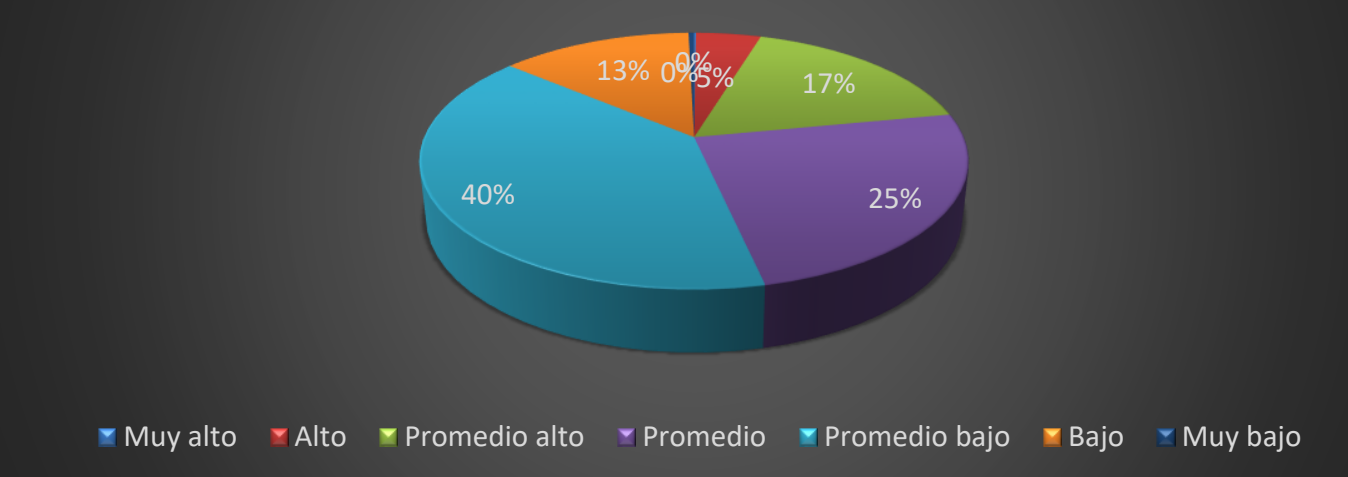

\section{Figura 3}

Comentario: La habilidad social en su dimensión toma de decisiones, se puede observar que el mayor porcentaje es $40 \%$ que significa una toma de decisiones de nivel promedio bajo, el $25 \%$ de los estudiantes representa una toma de decisiones de nivel promedio, el $17 \%$ de los estudiantes representa una toma de decisiones de nivel promedio alto, el $05 \%$ de los estudiantes representa una toma de decisiones de nivel alto y el nivel de toma de decisiones bajo el $13 \%$ de los estudiantes

\section{Tabla $\mathbf{N}^{\circ} 4$}

Frecuencia dimensiones comunicación

\begin{tabular}{ll}
\hline Niveles & Frecuencia \\
\hline 1. Muy bajo (Menor a 19) & 01 \\
2. Bajo (19 a 24) & 39 \\
3. Promedio bajo (25 a 29) & 173 \\
4. Promedio (30 a 32) & 198 \\
5. Promedio alto (33 a 35) & 177 \\
6. Alto (36 a 39) & 172 \\
7. Muy Alto (40 a mas) & 05 \\
\hline
\end{tabular}

- En la frecuencia de todos los factores, se puede observar la habilidad social en su dimensión comunicación la mayor cantidad de estudiantes está representado por 198 promedio, siguiente un nivel promedio alto 177 , el nivel promedio alto 172 , el nivel muy alto 05 , el nivel promedio bajo 173 , el nivel bajo 39 y el nivel muy bajo con 01 estudiante.

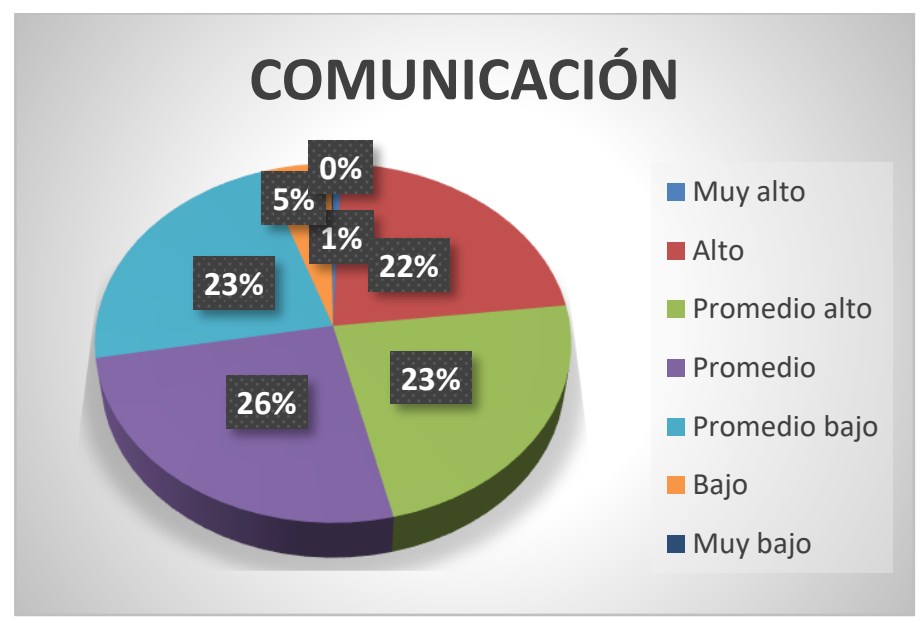

Figura 4 
Comentario: La habilidad social en su dimensión comunicación, se puede observar que el mayor porcentaje es $26 \%$ que significa una comunicación de nivel promedio, el $23 \%$ de los estudiantes representa una comunicación de nivel promedio alto, el $22 \%$ de los estudiantes representa una comunicación de nivel alto, el $01 \%$ de los estudiantes representa una comunicación de nivel muy alto, el $23 \%$ de los estudiantes representa una comunicación de nivel promedio bajo y el nivel de comunicación bajo el $05 \%$ de los estudiantes.

\section{Tabla $\mathbf{N}^{\circ} 5$}

Frecuencia dimensión Asertividad

\begin{tabular}{ll} 
Niveles & Frecuencia \\
\hline 1. Muy bajo (Menor a 21) & 01 \\
2. Bajo (21 a 34) & 19 \\
3. Promedio bajo (35 a 41) & 166 \\
4. Promedio (42 a 46) & 218 \\
5. Promedio alto (47 a 50) & 172 \\
6. Alto (51 a 54) & 185 \\
7. Muy Alto (55 a mas) & 04 \\
\hline
\end{tabular}

- En la frecuencia de todos los factores, se puede observar la habilidad social en su dimensión comunicación la mayor cantidad de estudiantes está representado por 218 promedio, siguiente un nivel promedio alto 172 , el nivel promedio alto 172 , el nivel alto 185 , el nivel muy alto 04 , el nivel bajo 19 y el nivel muy bajo con 01 estudiante

\section{Frecuencia dimensión Asertividad}

\section{ASERTIVIDAD}

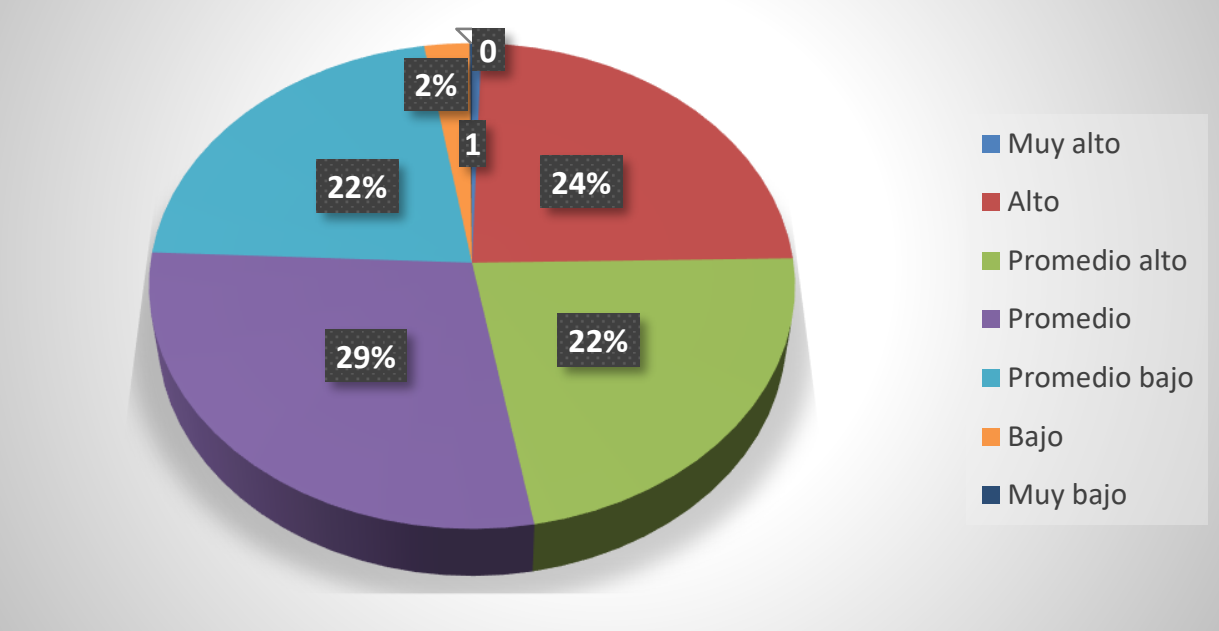

Figura 5

Comentario: La habilidad social en su dimensión asertividad, se puede observar que el mayor porcentaje es $26 \%$ que significa un asertividad de nivel promedio, el $23 \%$ de los estudiantes representa un asertividad de nivel promedio alto, el $22 \%$ de los estudiantes representa un asertividad de nivel alto, el $01 \%$ de los estudiantes representa un asertividad de nivel muy alto, el $23 \%$ de los estudiantes representa un asertividad de nivel promedio bajo y el nivel de asertividad bajo el $05 \%$ de los estudiantes.

Se puede observar que, en la variable de habilidad social, presentan un promedio de habilidad social inadecuada 162,2 como se observa en el cuadro estadístico de la muestra, lo que significa según este nivel, que los estudiantes presentan una habilidad social promedio bajo. 
Journal of the Academy Número 2 Enero-Junio 2020 - Pag. 18

En la figura 07 se puede observar la habilidad social en su dimensión comunicación la mayor cantidad de estudiantes está representada por 198 promedio, siguiente un nivel promedio alto 177, el nivel promedio alto 172 , el nivel muy alto 05 , el nivel promedio bajo 173 , el nivel bajo 39 y el nivel muy bajo con 01 estudiante.

Por otra parte, se puede observar la habilidad social en su dimensión comunicación la mayor cantidad de estudiantes está representada por 218 promedio, siguiente un nivel promedio alto 172, el nivel promedio alto 172 , el nivel alto 185, el nivel muy alto 04 , el nivel bajo 19 y el nivel muy bajo con 01 estudiante.

Los resultados en la figura $\mathrm{N}^{\circ} 06$ en la dimensión toma de decisiones se puede observar la habilidad social en su dimensión toma de decisiones la mayor cantidad de estudiantes está representado por 303 promedio bajo, siguiente un nivel promedio 188, el nivel promedio alto 132, el nivel bajo 103 y el nivel muy bajo con 03 estudiantes, En virtud de los resultados se puede decir que los alumnos no presentan un buen nivel de interacción social, lo que reflejan en las malas toma de decisiones.

En la frecuencia de todos los factores, se puede observar la habilidad social en su dimensión autoestima la mayor cantidad de estudiantes está representado por 266 promedio bajo, siguiente un nivel promedio 187, el nivel promedio alto 132, el nivel bajo 97 y el nivel muy bajo con 08 estudiantes

\section{Conclusiones}

Habiendo analizado y discutido los resultados de los cuatro objetivos planteados, donde se buscó identificar el nivel de habilidad social de los estudiantes de la Institución Educativa Agropecuario 08 con Jornada Escolar Completa en sus cuatro dimensiones se llega a la conclusión.

El nivel de habilidad social en su dimensión "toma de decisiones", la mayoría de estudiantes tienen un nivel de toma de decisiones promedio bajo, la cual demuestra que los estudiantes tienen habilidades sociales inadecuadas.

En virtud de los resultados se puede decir que los alumnos no presentan un buen nivel de interacción social, ya que dentro de la Autoestima de los 765 estudiantes posee un nivel bajo de Autoestima 97, lo que se refleja en las malas relaciones interpersonales.

El nivel de habilidad social en su dimensión "comunicación", la mayoría de estudiantes tienen un nivel de comunicación promedio alto que son el $23 \%$, en la cual demuestra que los estudiantes desarrollan un buen nivel promedio de comunicación entre sus compañeros.

Referido a las otras dimensiones, se requiere desarrollar actividades dirigidas a estos estudiantes a fin de favorecer y mejorar el nivel de habilidades sociales teniendo como resultado general de un $48 \%$ de los estudiantes se encuentra con un nivel de habilidad social inadecuada.

\section{Referencias bibliográficas}

Arkowitz, H. (1981). Assessment of social Skills en M Hersen y Bellakc, a practical handbook. Nueva York, Pergamon.

Branden, Nathaniel. (1998). "Los seis pilares de la autoestima" Buenos Aires: Paidos Iberoamérica.

lannizzotto, M. E. (2009). Hacia una genuina valoración del sí mismo de la persona. Información Filosófica, VI (13), 79- 110. doi:10.3308/2009.01 
Journal of the Academy Número 2 Enero-Junio 2020 - Pag. 19

Hernández, Fernández y Bapista. (2006) Metodología de la investigación. Ed Mc Graw hill.

Sánchez C. H. \& Reyes R, C. (1994). Grandes personajes de la psicología contemporánea Lima: Facultad de Psicología. Universidad Ricardo palma recuperado de http://www.urp.edu.pe/pdf/psicologia/revista03.pdf

Sánchez C. H. \& Reyes R, C. (2006) Desarrollo de una escala factorial para medir la felicidad. Revista Interamericana de Psicología. Recuperado de http://www.urp.edu.pe/pdf/psicologia/revista-025pdf

Emmons y Alberti R. (1983). Failure, Winning at the losing game in assertiveness traing, Failures, Nueva York, Wiley. 